\title{
3D-FLAIR imaging with optimized scan parameters to visualize endolymphatic hydrops with intravenous gadolinium-based contrast media
}

\author{
Jinye $\mathrm{Li}^{1}$, Lixin $\mathrm{Sun}^{1}$, Long $\mathrm{Li}^{1}$, Gesheng Song ${ }^{2}$, Han $\mathrm{Xu}^{3}, \mathrm{Na} \mathrm{Hu}{ }^{4}$, Hui Zhao ${ }^{4}$, Jing Tian ${ }^{4}$, \\ Ting $\mathrm{Xu}^{4}$, Weiqiang Dou ${ }^{5}$, Ruozhen Gong ${ }^{6}$, and Chuanting $\mathrm{Li}^{6}$ \\ ${ }^{1}$ Shandong Provincial ENT HospitalCheeloo College of MedicineShandong University \\ ${ }^{2}$ Shandong province Qianfoshan Hospital \\ ${ }^{3}$ Shandong Provincial HospitalCheeloo College of MedicineShandong University, \\ ${ }^{4}$ Shandong Provincial ENT HospitalCheeloo College of MedicineShandong University, \\ ${ }^{5} \mathrm{GE}$ Healthcare, MR Research China \\ ${ }^{6}$ Shandong Medical Imaging Research Institute, Cheeloo College of MedicineShandong \\ University
}

June 24, 2020

\begin{abstract}
Objective: To explore the optimal imaging parameters, including the optimal scan time and scan angle, of 3D-FLAIR imaging in labyrinthine for patients with vertigo and sensorineural hearing loss. Design: A prospective clinical study. Setting: Academictertiary center. Participants: Twenty-two patients with unilateral vertigo and sensorineural hearing loss were prospectively reviewed between July 2018 and June 2019. Main outcome measures: The corresponding signal-intensity-ratios and contrastto-noise-ratios of these images acquired at different time points after the administration of gadolinium contrast agent at double dose were obtained on the 3D-FLAIR images. The scan angles, aiming to obtain the relative maximum areas of the saccule, utricle and lateral semicircular canal displayed at the same level, were also evaluated with the anterior skull base in the sagittal position. Results:3D-FLAIR images with double-dose injection of gadolinium contrast acquired at 6 hours post-injection showed the strongest image contrast in the cochlea compared to those at 4 and 8 hours (affected ear: $2.05 \pm 0.72$ vs $1.85 \pm 0.76$ vs $1.79 \pm 0.60$, unaffected ear: $1.97 \pm 0.68$ vs $1.59 \pm 0.28$ vs $1.57 \pm 0.31$ ). The significantly higher CNR values were shown at 6 hours in both the affected and unaffected ears than those acquire at other time points $(\mathrm{P}<0.05)$. In addition, the optimal angles ranging from 6.20 to 13.6 degrees (mean:10.74 \pm 2.24 degrees; $\mathrm{P}<0.01$ ) were obtained. Conclusion: The optimal scan time was found at 6 hours after injecting contrast agent at double-dose. Together with the best scan angle obtained, the EH can be accurately assessed on 3D-FLAIR images.
\end{abstract}

\section{Key points}

- A double-dose injection $(0.4 \mathrm{ml} / \mathrm{kg})$ has started being applied in clinic nowadays. The optimal image contrast was however, not observed after 4 hours post a double-dose injection. Hence, it is crucial to investigate the optimal scan time at double dose administration.

- We found that 3D-FLAIR images with double-dose injection of gadolinium contrast acquired at 6 hours post-injection showed the strongest image contrast in the cochlea. The significantly higher CNR values were shown at 6 hours than those acquire at other time points. In addition, the optimal angles ranging from 6.20to 13.6 degrees were obtained. 
- Therefore, the optimal scan time was found at 6 hours after injecting contrast agent at double-dose. Together with the best scan angle obtained, the EH can be accurately assessed on 3D-FLAIR images.

\section{Introduction}

Ménière's disease(MD)is characterized by three clinical symptoms of fluctuating low-frequency sensorineural hearing loss, tinnitus and/or aural fullness, and vertigo attacks in about $0.2-0.5 \%$ of the general population $(1,2)$. The diagnosis of MD is based upon the typical clinical syndrome complemented with a battery of audiological, vestibular, and electrophysiological tests and the demonstration of endolymphatic hydrops $(\mathrm{EH})(3,4)$. However, the latter onewas lack of a gold standard diagnostic test, and could only be obtained by histological post-mortem examination previously.

Magnetic resonance imaging(MRI) has been reported for the potential in the clinical diagnosis of MD.After Zou et al. was the first study reportinga separate visualization of endolymph and perilymph regions in living humans using MRI (5), Nakashima et al (6)showed the feasibility of gadolinium enhanced MRI in the visualization of $\mathrm{EH}$ in living MD patients. With intravenous injection of gadolinium-based contrast media (GBCM), three-dimensional fluid-attenuated-inversion-recovery (3D-FLAIR) imaging hasbeen reported to visualize EH (7-11). The optimal scan time reported was 4hours after the administration of GBCM at a single-dose $(0.2 \mathrm{ml} / \mathrm{kg})(7)$. The concentration of GBCM in the perilymph after single-dose intravenous injection however is too low to be detected by convention 3D-FLAIR in patients without impaired permeability of the blood labyrinthine barrier (12)and is challenging to achieve consistent gadolinium enhancement in the upper part of the cochlea $(1,13)$, although it has been used widely in a number of clinical applications. Therefore, a higher dose of contrast agent is requested in labyrinthineimaging. Under FDA approved maximal triple dose of gadolinium (i.e. $0.3 \mathrm{mmol} / \mathrm{kg}$ ) for human imaging, a double-dose injection $(0.4 \mathrm{ml} / \mathrm{kg}$ ), aiming to obtain higher signal intensity (14), has started being applied in clinic nowadays. The optimal image contrast was however, not observed after 4 hours posta double-dose injection. Hence, it is crucial to investigate the optimal scan time at double dose administration.

On the other hand, the 3D image volume for bilateral inner ear is essential to be adjusted in three directions. While a proper adjustment in axial and coronal directions can ensure symmetrical display for bilateral inner ears, a suitable scan angle applied in sagittal view plays an important role in labyrinthine imaging. With this, the relative maximum areas of the saccule, utricle and lateral semicircular canal can thus be provided at the same level to accurately estimate the EHand judge whether EH herniation occurs in the lateral semicircular canal. However, this optimal scan angle with the anterior skull base remains unknow so far.

Therefore, this study wasto explore the optimal imaging parameters of 3D-FLAIR, including the optimal scan time at double dose administration and scan angle, in labyrinthine imaging for patients with vertigo and sensorineural hearing loss.

\section{Materials and methods}

\section{Ethical consideration}

This study was approved by the Hospital Research Ethics of Committee of [removed for blind peer-review] Provincial ENT Hospital affiliated to [removed for blind peer-review] University .

\section{Subjects}

This prospective clinical studyincluded 22 patients (48+-13 years old) with unilateral vertigo and sensorineural hearing loss, who visited our outpatient department from July 2018 to June 2019. All patients gave written consent. The inclusion criteria were defined as follows: patients with unilateralvertigo and sensorineural hearing loss, and an age above 18 years. The exclusion criteria werealso applied including: (1) physical trauma or head and neck neoplasm, brainstem disease.(2) history of chronic inflammatory or suppurative ear disease, prior ear surgery of any kind. (3) serious psychiatric disease, haematological disease, prior treatment with chemotherapy agents or other immunosuppressive drugs. (4) MR-related contraindications, a history of allergies to GBCM and an inability to provide informed consent, as well as middle ear 
pathology that could impede local contrast uptake. All patients underwentdouble-dose intravenous injection of ionic gadobenatedimeglumine (Gd-BOPTA; MultiHance, Bracco Pharmaceutical Co. Ltd., Shanghai, China, $0.4 \mathrm{~mL} / \mathrm{kg}$ ).

For each patient, 3D-FLAIR images were acquired four, six and eight hours after the agent injection. An extra image acquisition was implemented for eleven patients of them at 10 hours after agent injection. No patients were excluded from the study due to insufficient image quality.

\section{MR experiments}

All experiments were performed on a 3T clinical scanner (Discovery 750w, GE Healthcare, USA) equipped with an 8-channel phased-array coil. A 3D-FLAIR sequence was employed for labyrinthineimaging with scan parameters of repetition time $=9000 \mathrm{~ms}$; echo time $=$ aximum $(128 \mathrm{~ms}-132 \mathrm{~ms})$; inversion time $=2500 \mathrm{~ms}$; echotrainlength $=140$; bandwidth $=36 \mathrm{Khz}$; matrix size $=256 \times 256 ; \mathrm{NEX}=2$; slice thickness $=1.6 \mathrm{~mm}$, and fieldofview $=21 \mathrm{~cm} \times 16 \mathrm{~cm}$. The scan time was 5 minutes 46 seconds.

\section{Data analysis}

All data were processed at a vendor-provided ADW 4.6 workstation (GE Medical Systems).

To evaluate the contrast enhancement in the cochlear fluid quantitatively, regions of interest (ROI)s were separately set manually in the basal turn of the cochlea, brainstem and cerebrospinal fluid space of the cerebellopontine angleon 3D-FLAIR images for all patients (Fig.1). The SIR was calculated using the following equation:

$\mathrm{SIR}=\mathrm{SI}_{\text {cochlea }} / \mathrm{SI}$ brainstem $($ Eq.1)

, where $\mathrm{SI}_{\text {cochlea }}$ represents the signal intensity of the cochlear basal turn, $\mathrm{SI}_{\text {brainstem }}$ is the brainstem. Additionally, the signal intensities in the bilateral cochlear regions (i.e., with and without affection) were, respectively, evaluated using a defined CNR equation:

$\mathrm{CNR}=\left(\mathrm{SI}_{\text {cochlea }}-\mathrm{SI}_{\text {cerebrospinal fluid }}\right) / \sigma_{\text {brainstem }}(\mathrm{Eq} .2)$

, where $\mathrm{SI}_{\text {cochlea }}$ represents the signal intensity of cochlear basal turn, $\mathrm{SI}_{\text {cerebrospinal fluid }}$ is the cerebrospinal fluid space, and $\sigma_{\text {brainstem }}$ is the standard deviation of the signal intensity of the brainstem.

To obtain the optimal scan angle, the anterior skull base was used as the baseline in sagittal direction (zero degree). The 3D-FLAIR images of 22 patients were reconstructed so that the relative maximum areas of the saccule, utricle and lateral semicircular canal can be displayed at the same level. The MR images were qualitatively analyzed twice by two experienced head and neck radiologists blinded to the clinical findings. In total,six measurements were performed and the mean level was calculated.

The degree of $\mathrm{EH}$ in the vestibule and cochlea was assessed by visual comparison of the relative areas of the non-enhanced endolymphatic space versus the contrast-enhanced perilymph space on 3D-FLAIR at 6 hours post-injectionin the axial plane, separately for the cochlea and the vestibule. The degree of cochlear hydrops was categorized as none, grade I, or grade II according to the criteria previously described by Baráth et al (15).

However, to evaluate the degree of the vestibular hydrops, we used the four-stage grading system by Bernaerts et al. (16).Vestibular EH and cochlear EH were reviewed twice by two experienced readers.

The SIR and CNR values were systematically compared between images acquired at different post-injection time using a Student's t-test in SPSS 20.0 (IBM, Chicago, IL). The Student's t-test was also applied to estimate the difference of the relative maximum areas of the saccule, utricle and lateral semicircular canal at different scan degrees applied. Additionally, the Cohen's kappa statistic was used to estimate the interobserver agreement on detecting and grading EH. The significance threshold was set as $P=0.05$.

\section{Results}


With double-dose injection, the 3D-FLAIR images acquired at 6 hours post-injection showed the strongest image contrast in the cochlea of 44 ears for twenty-two patients (Fig.2). The SIR and CNR values were separately found in the cochlea of the affected 22 ears 4 hours, 6 hours, 8 hours and 10 hours after agent injection (Table 1). Comparing the SIR and CNR values between 4 hours and 6 hours, and between 6 hours and 8 hours, respectively, significantly higher values were shown at 6 hours in both the affected and unaffected ears $(P<0.05)$.

The optimal angles with the anterior skull base in sagittal view were obtained, ranging from 6.20to13.6 degrees (mean:10.74 \pm 2.24 degrees) and being parallel to the gyrus rectusnearly. The mean level of 10.74 degreecan thus be considered the optimal scan angle from the anterior skull base (Fig.3a,b), if compared to the scanning in parallel to the anterior skull base $(P<0.01$; Fig. 3 c,d).

Cochlear hydrops was present in 14affectedears (grade I in 10 and grade II in 4 cases), and vestibular hydrops was detected in 17affectedears (grade I in 3, grade II in 10 and grade III in 4 instances). Cohen $\mathrm{K}$ test for interobserver agreement was 0.97 for cochlear findings and 0.98 for the vestibule (normal and abnormal).Seventy-seven percent (17/22) of clinically diseased ears had EH shown by MR imaging, whereas $9 \%(2 / 22)$ of clinically normal ears showed $\mathrm{EH}$ with MR imaging.

\section{Discussion}

In this study, we explored the optimal imaging parameters, including the optimal scan time and scan angle, of 3D-FLAIR in labyrinthine imaging for patients with vertigo and sensorineural hearing loss. The optimal scan time was found at 6 hours after injecting the contrast agent at double-dose and the best scan angle ranged from 6.20 to13.6 degrees (mean:10.74 \pm 2.24 degrees) was also obtained.

As the first main finding, we found that 3D-FLAIR can visualize endolymphatic hydrops 4 hours, 6 hours, 8 hours and 10 hours after double dose administration, and the significantly higher CNR and SIR values were shown at 6 hours in both the affected and unaffected ears. As the CNR value reflects the signal difference between the endolymphatic and perilymphatic regions, a significantly higher CNR in 3D-FLAIR images at 6 hours allowed a more robust distinguishing between the endolymphatic and perilymphatic regions if compared with the images acquired at other time points. That is to say, 6 hours after injecting the contrast agent can be considered an optimal scan time at double-dose. Nakashima et al (7) performed MR imaging 4 $\mathrm{h}$ after intravenous injection of a double dose of gadolinium in patients with Ménière's disease at 3T. This findingwas however different fromthe 4 hours at a single-dose injection reported previously (17). The reason might be explained that similar to that the extent of cochlear enhancement varied with the concentration of the drug (6), the peak time of the drug concentration in the cochlea also varied.

Additionally, the obtained optimal scan angle of 3D-FLAIRwas about 10.74 \pm 2.24 degrees from the anterior skull basein sagittal view. With this, the relative maximum areas of the saccule, utricle and lateral semicircular canal can be displayed at the same level. As the bilateral semicircular canals are fully visible at the same level, we can visualize the saccule and utricle simultaneously, and judge whether EH herniation occurs in the lateral semicircular canal.3D images of the membranous labyrinth directly reconstructed from temporal bone sections suggest that in EH changes of saccular morphology are more sensitive than those of the utricle (18). Accordingly, Attyé et al. described the inversion of the saccule-to-utricle area ratio (SURI) as a qualitative marker (19). In addition, the vestibular semiquantitative, three-stage EH grading system proposed byBaráth et al. (15)and four-stage EH grading system proposed by Bernaerts et al. (16) were commonly used.With these semi-quantitative MR grading systems, the area of the utricle and saccule is requested to be obtained. It is thus important to display the relative maximum area of both at the same level.Besides these, Gurkov et al. (20)reported that EH herniation into the lateral semicircular canal (SCC) seemed to have a morphological correlation of an impaired caloric response in patients with MD.Therefore, it is beneficial to observe the degree of EH by simultaneously displaying the lateral SCC on the same level. When scanning the inner ear, we routinely place the scan line parallel to the lateral SCCbased on the image at coronal view.The scanning angle needed to be adjusted appropriately on the sagittal image, aiming to show the largest relative areas of the utricle and saccule, and lateral SCC on the same horizontal plane. 
Different scan angles in the sagittal positioning phase show different relative areas of the utricle and saccule, and lateral SCC accordingly. Therefore, a proper scanning angle is very important.The optimal scan time and scan angle can help us to accurately determine EH.

By histopathology, Rauch et al. (21) and Merchant et al. (22)found EH in 5\% and $26 \%$ of temporal bones in patients without previous symptoms of MD. The former figure corresponds to an incidence of $9 \%$ in clinically "silent" ears in our series. EH was found on the clinically affected side in 17/22 ears (77\%). Histopathologically, Fraysse et al. (23) described results in $93 \%$ of 21 affected ears.Our rate of EH is slightly lower than the previous ones, because the patients we included were not all MD patients. All nine definite MD patients (4) included showed EH (100\%). The rate is higher than the results obtained in the former study (23).

One major limitation of this study is that the contralateral normal ears of the vertiginous patients were used as controls. Due to ethical reasons it is not allowed to observethe peak time of the drug concentration in the cochleaof a healthy populationwith gadolinium injection.

\section{Conclusion}

In summary, in this study the optimal scan time was found at 6 hours after injecting the contrast agent at double-dose, and the optimal scan angle was about $10.74 \pm 2.24$ degrees from the anterior skull base.With the optimal scan time and scan angleapplied, the EH on 3D-FLAIR imaging can be accurately estimated.

\section{Data Availability Statement:}

All data generated or analyzed during this study are included in this article.

\section{References}

1.Havia M, Kentala E, Pyykko I. Prevalence of Ménière's disease in general population of Southern Finland. Otolaryngol Head Neck Surg. 2005;133(5):762-768.

2. Alexander TH, Harris JP. Current epidemiology of Ménière's syndrome.Otolaryngol Clin North Am. 2010; 43(5):965-970.

3. Nakashima T, Pyykkö I, Arroll MA, et al.Ménière's disease. Nat Rev Dis Primers. 2016; 2:16028.

4. Lopez-Escamez JA, Carey J, Chung WH, et al. Diagnostic criteria for Menière's disease. J Vestib Res. 2015; 25(1):1-7.

5. Zou J, Pyykko I, Bjelke B, et al. Communication between the perilymphaticscalae and spiral ligament visualized by in vivo MRI.Audiol Neuro-Otol . 2005;10(3):145-152.

6. Nakashima T, Naganawa S, Sugiura M, et al. Visualization of endolymphatic hydrops in patients with Ménière'sdisease.Laryngoscope .2007; 117(3):415-420.

7. Nakashima T, Naganawa S, Teranishis M, et al.Endolymphatic hydrops revealed by intravenous gadolinium injection in patients with Ménière's disease. Acta Oto-Laryngologica. 2010;130(3): 338-343.

8.Naganawa S, Yamazaki M, Kawai H, et al. Visualization of endolymphatic hydrops in Ménière's disease with single-dose intravenous gadolinium-based contrast media using heavily $\mathrm{T}_{2}$-weighted 3D-FLAIR.MagnReson Med Sci. 2010;9(4):237-242.

9.Sugimoto S, Yoshida T, Teranishi M, et al. The relationship between endolymphatic hydrops in the vestibule and low-frequency air-bone gaps.Laryngoscope. 2018;128(7):1658-1662.

10. Yoshida T, Sugimoto S, Teranishi M, et al.Imaging of the endolymphatic space in patients with Ménière's disease. Auris Nasus Larynx .2018;45(1):33-38.

11. Morimoto K, Yoshida T, Sugiura S, et al.Endolymphatic hydrops in patients with unilateral and bilateral Ménière's disease. Acta Otolaryngol. 2017;137(1):23-28. 
12. Tagaya M, Teranishi M, Naganawa S, et al. 3 Tesla magnetic resonance imaging obtained 4 hours after intravenous gadolinium injection in patients with sudden deafness.Acta Otolaryngol. 2010;130(6):665-669.

13. Carfrae MJ, Holtzman A, Eames F, et al.3 tesla delayed contrast magnetic resonance imaging evaluation of Ménière's disease.Laryngoscope. 2008;118(3):501-505.

14.Niyazov DM, Andrews JC, Strelioff D, et al. Diagnosis of

endolymphatic hydrops in vivo with magnetic resonance imaging. OtolNeurotol. 2001;22(6):813-817.

15. Baráth K, Schuknecht B, Naldi AM, et al. Detection and grading of endolymphatic hydrops in Menière disease using MR imaging. AJNR Am J Neuroradiol. 2014;35(7):1387-1392.

16.Bernaerts A, Vanspauwen R, Blaivie C, et al. The value of four stage vestibular hydrops grading and asymmetric perilymphatic enhancement in the diagnosis of Menière's disease on MRI.Neuroradiology. 2019;61(4):421-429.

17.Naganawa S, Komada T, Fukatsu H, et al. Observation of contrast enhancement in the cochlear fluid space of healthy subjects using a 3D-FLAIR sequence at 3 Tesla. Eur Radiol. 2006;16(3):733-737.

18. Morita N, Kariya S, FarajzadehDeroee A, et al. Membranous labyrinth volumes in normal ears and Ménière disease: a threedimensional reconstruction study. Laryngoscope. 2009;119(11):2216-2220.

19.Attyé A, Eliezer M, Boudiaf N, et al. MRI of endolymphatic hydrops in patients with Ménière's disease: a case-controlled study with a simplified classification based on saccular morphology.Eur Radiol. 2017;27(8):3138-3146.

20. Gurkov R, Flatz W, Louza J, et al. Herniation of the membranous labyrinth into the horizontal semicircular canal is correlated with impaired caloric response in Ménière's disease. OtolNeurotol. 2012;33(8):13751379 .

21. Rauch SD, Merchant SN, Thedinger BA. Ménière's syndrome and endolymphatic hydrops: double-blind temporal bone study. Ann OtolRhinolLaryngol .1989;98(11):873-83.

22. Merchant SN, Adams JC, Nadol JB Jr. Pathophysiology of Ménière's syndrome: are symptoms caused by endolymphatic hydrops? OtolNeurotol. 2005;26(1):74-81.

23.Fraysse BG, Alonso A, House WF. Ménière's disease and endolymphatic hydrops: clinicalhistopathological correlations. Ann OtolRhinolLaryngol Suppl .1980;89 (6 Pt 3):2-22.

\section{Figure Legends}

Fig. 1 An example of the region of interest (ROI) setting on a contrast-enhanced 3D-FLAIR image. The ROI (the area ofROI $=1 \mathrm{~mm}^{2}$ ) for the cochlear fluid signal intensity measurement is manually drawn around the basal turn of the cochlea. Circular ROIs for the signal intensity measurement of the brainstem (the area ofROI $=4 \mathrm{~mm}^{2}$ ) and the cerebellopontine angle (the area ofROI $=4 \mathrm{~mm}^{2}$ ) are set in the most artefact-free area of the brainstem center and the ipsilateral cerebellopontine angle.

Fig. 2 A 39-year-old male Ménière's disease. Fig. a 3D-FLAIR images at the left cochlear basal turn level were obtained $4 \mathrm{~h}, 6 \mathrm{~h}, 8 \mathrm{~h}$ and $10 \mathrm{~h}$ after the administration of GBCM. Signal intensity of the cochlear fluid in the basal turn is highest on the images obtained $6 \mathrm{~h}$ after contrast administration (arrows). Fig. bThe signal intensity ratio between the cochlea and the brainstem on 3D-FLAIR image were plotted for the patient. The signal intensity ratio (SIR) was highest at $6 \mathrm{~h}$ after contrast administration.

Fig. 3 A 52-year-old male Ménière's disease. Fig. aThescan angle with the anterior skull base in the sagittal position was 10.8 degrees. When scanned at this angle, we get Fig. b. Fig. b The 3D FLAIR image display the relative maximum areas of the saccule (solid arrows), utricle (dotted arrows) and the lateral semicircular canal (arrowheads) on the same level. Fig. cThescan angle with the anterior skull base in the sagittal position was 0 degrees. When scanned at this angle, we get Fig. d. Fig. d The 3D FLAIR image display the saccule 
(solid arrows), utricle (dotted arrows), but the posterior part of the lateral semicircular canal (arrowheads) is not shown on the same level simultaneously.

\section{Hosted file}

Figures.docx available at https://authorea.com/users/336561/articles/462221-3d-flairimaging-with-optimized-scan-parameters-to-visualize-endolymphatic-hydrops-withintravenous-gadolinium-based-contrast-media

\section{Hosted file}

Table.docx available at https://authorea.com/users/336561/articles/462221-3d-flair-imagingwith-optimized-scan-parameters-to-visualize-endolymphatic-hydrops-with-intravenousgadolinium-based-contrast-media 